\title{
Platelet adhesiveness: the effect of centrifugation on the measurement of adhesiveness in platelet-rich plasma
}

\author{
J. A. MCBRIDE 1 \\ From the Department of Haematology, Royal Postgraduate Medical School, London
}

SYNOPSIS Platelet adhesiveness has been measured in citrated whole blood and in platelet-rich plasma obtained from normal subjects, splenectomized patients, and from patients in whom the diagnosis of recurrent venous thrombosis had been made. The duration of centrifugation used in the preparation of platelet-rich plasma was found to have a profound effect on the measurement of platelet adhesiveness because the figure for platelet adhesiveness measured in platelet-rich plasma obtained by centrifugation was considerably lower than that found in citrated whole blood. This effect was particularly marked when platelet-rich plasma was obtained from subjects in whom platelet adhesiveness measured in whole blood was increased.

Platelet adhesiveness measured by an in vitro technique is currently of considerable interest. Most studies of platelet adhesiveness have been carried out in whole blood and have utilized the contact of platelets with a glass surface either in a rotating bulb (Wright, 1941) or standard column of glass beads (Hellem, 1960). However, recently a number of studies have been carried out in which adhesiveness has been measured in platelet-rich plasma (Hellem, 1960; O'Brien, 1961; Caspary and Peberdy, 1965). Few studies have been reported comparing one whole blood method with another (Horlick, 1961; Reber and Studer, 1965; Hirsh, McBride, and Wright, 1966) and only one in which platelet adhesiveness was measured both in whole blood and platelet-rich plasma (Cronberg, Nilsson, and Silwer, 1966). The present study was undertaken to compare adhesiveness measured in whole blood and plateletrich plasma under varying experimental conditions, using a modification (Hirsh and McBride, 1965) of the glass bead column technique described by Hellem (1960).

\section{MATERIALS}

Glass beads (Reflex Perlen), $0.5 \mathrm{~mm}$ in diameter, were prepared as described by Hellem (1960). Portex tubing N.T. $13,0.55 \mathrm{~cm}$ internal diameter, was used in the

${ }^{1}$ In receipt of a grant from Glaxo Ltd.

Received for publication 30 August 1967. preparation of the columns which contained $2.5 \mathrm{~g}$ of glass beads. Adenosine diphosphate (ADP) (Sigma Chemicals) was dissolved in buffered saline and stored in small aliquots at $-20^{\circ} \mathrm{C}$. This solution was thawed and diluted immediately before use.

\section{METHODS}

Blood, $40 \mathrm{ml}$, was collected in a disposable plastic syringe. Aliquots were delivered into six plastic tubes containing $3.1 \%(w / v)$ trisodium citrate $(9$ parts of blood to 1 part of citrate). Platelet adhesiveness was measured in citrated whole blood after the first tube had been allowed to stand at room temperature $\left(19-21^{\circ} \mathrm{C}\right)$ for 60 minutes. After gentle mixing $2 \mathrm{ml}$ of citrated blood was passed at a constant rate through a standard column $(6 \mathrm{~cm}$ in length, containing $2.5 \mathrm{~g}$ glass beads) by means of a motor-driven $2 \mathrm{ml}$ disposable plastic syringe. The advancing head of the column of blood was in contact with the glass beads for $26 \pm 1 \mathrm{sec}$ and the contents of the syringe had been delivered into the column in 90 seconds.

The second tube was allowed to stand undisturbed on the bench to allow the red cells to sediment for 60 minutes. At the end of this time the supernatant plasma was carefully removed. This plasma is subsequently referred to as ' $\mathrm{O}$ ' minutes of centrifugation (although the platelets had been subjected to $1 \mathrm{~g}$ for 60 minutes). The third, fourth, fifth, and sixth tubes were centrifuged at $200 \mathrm{~g}$ in a MSE Major centrifuge for four, eight, 12, and $16 \mathrm{~min}$, respectively. After centrifugation the supernatant plasma was carefully removed. Care was taken to see that the buffy coat layer was not disturbed. When all five plasma samples had been obtained platelet adhesiveness 
was measured. Of the ADP solution, $0.2 \mathrm{ml}$ was added to $1.8 \mathrm{ml}$ of platelet-rich plasma in a $2 \mathrm{ml}$ plastic syringe producing a final concentration of ADP of $0.1 \mu \mathrm{g}$ per millilitre plasma. The syringe was inverted six times then placed in the syringe drive. The motor of the syringe drive was started exactly $30 \mathrm{sec}$ after the addition of the ADP.

Platelet counts were performed before and after the passage of the blood or plasma through the column by the method of Brecher and Cronkite (1950). At least 500 platelets were counted in each counting chamber. The percentage decrease in the platelet count after passage through the column is taken to be a reflection of platelet adhesiveness. The other haematological methods used were those described by Dacie and Lewis (1963).

\section{PATIENTS STUDIED}

Platelet adhesiveness was measured in 10 normal subjects and in 14 patients who had increased platelet adhesiveness when it was measured in whole blood (Hirsh and McBride 1966). A diagnosis of recurrent venous thrombosis or pulmonary embolism had been made in four of these patients and 10 of them had undergone splenectomy. This had been carried out in the course of gastric or oesophageal surgery in seven and for the treatment of idiopathic thrombocytopenic purpura in three. All patients had normal packed cell volumes at the time of study.

\section{RESULTS}

PLATELET ADHESIVENESS IN NORMAL SUBJECTS The results of a typical experiment are shown in Figure 1. This shows that platelet adhesiveness measured in whole blood is approximately equal to adhesiveness measured in platelet-rich plasma providing that the plasma has been obtained by centrifuging the citrated whole blood for eight min or less. After 12 or 16 minutes' centrifugation the percentage adhesiveness measured in platelet-rich plasma was considerably lower than that found in whole blood. Data obtained from 10 similar experiments on blood obtained from haematologically normal subjects are summarized in Table I. There is no statistical difference between adhesiveness measured in whole blood

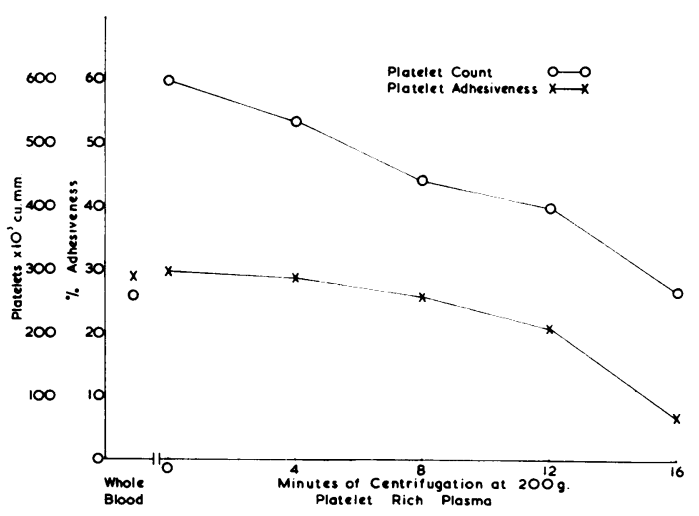

FIG. 1. The effect of the duration of centrifugation on the preparation of platelet-rich plasma on the platelet count and $\omega$ percentage platelet adhesiveness in a normal subject. The $\omega$ platelet count and percentage platelet adhesiveness $\underset{ }{V}$ measured in citrated whole blood are also shown.

and platelet-rich plasma which has been obtained by sedimentation at room temperature for one hour or $\vec{\theta}$ by centrifugation for four or eight min at $200 \mathrm{~g}$. $\mathscr{\odot}$ Platelet adhesiveness measured in platelet-rich plasma obtained by centrifugation for 12 minutes or more was significantly lower than that obtained in whole blood.

PLATELET COUNT IN PLATELET-RICH PLASMA IN $\stackrel{\varrho}{\circledR}$ NORMAL SUBJECTS The effects of centrifugation on $\overrightarrow{\vec{B}}$ the total platelet count and the number of adhesive 3 platelets in platelet-rich plasma are shown in Figure 2. The total platelet count in platelet-rich plasma after centrifugation is expressed as a percentage of the total platelet count in the plasma obtained by sedimentation at room temperature. Similarly, the number of adhesive platelets in each specimen is expressed as a percentage of the adhesive platelet count in the sedimented specimen. The mean values of the $\mathbf{1 0}$ observations are shown in Table I. It will

TABLE I

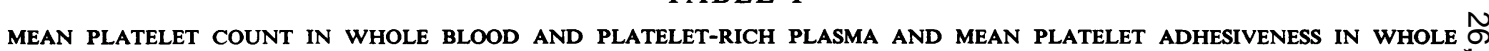
BLOOD AND PLATELET-RICH PLASMA OF 10 NORMAL SUBJECTS

Whole Blood Platelet-rich Plasma

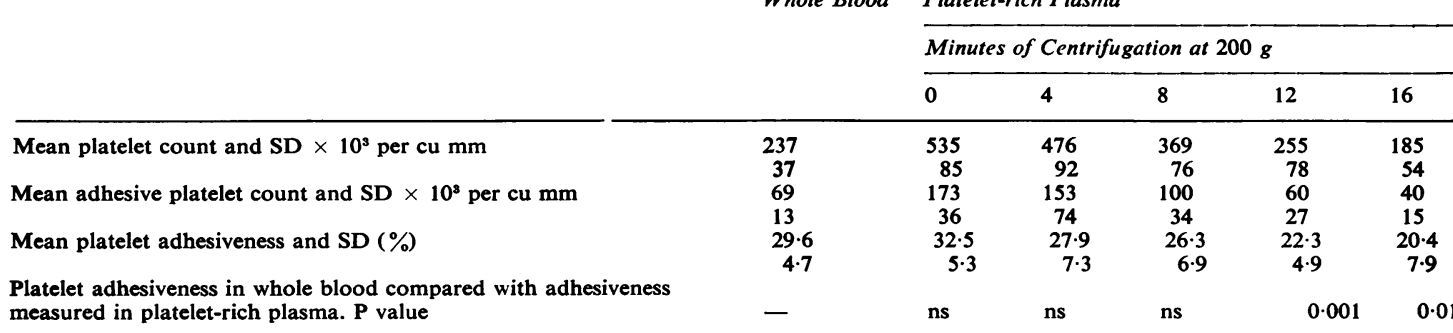

ns $=$ not significant. 


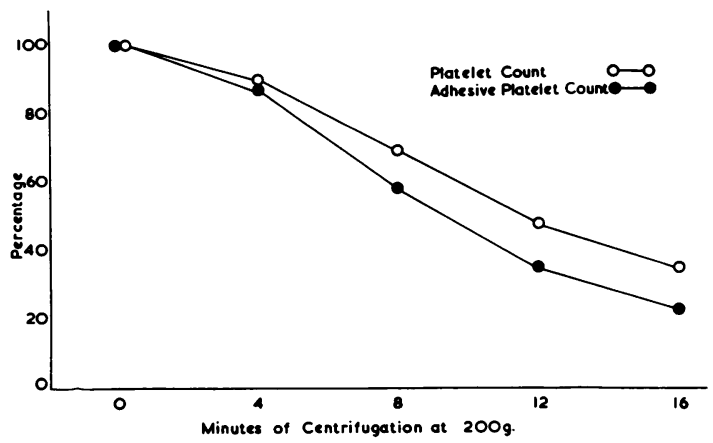

FIG. 2. The total platelet count and adhesive platelet count expressed as a percentage of those values found in plateletrich plasma obtained by sedimentation. The mean values obtained from 10 normal individuals are shown.

be seen that both the total platelet count and the number of adhesive platelets decreased at approximately the same rate (Fig. 2).

PLATELET ADHESIVENESS AFTER SPLENECTOMY Platelet adhesiveness measured in whole blood or in plateletrich plasma obtained by sedimentation is considerably increased after splenectomy. However, after only four min of centrifugation there is a considerable reduction when adhesiveness is measured in the plasma so obtained. The degree of reduction is statistically highly significant (Table II).

PLATELET COUNT AFTER SPLENECTOMY After splenectomy the rate at which platelets are removed from supernatant plasma by centrifugation is greatly increased (Figure 3). It would appear that adhesive platelets are removed more rapidly than nonadhesive platelets. After only four min of centrifugation the total platelet count was reduced by $52 \%$ while the adhesive platelet count was reduced by $74 \%$.

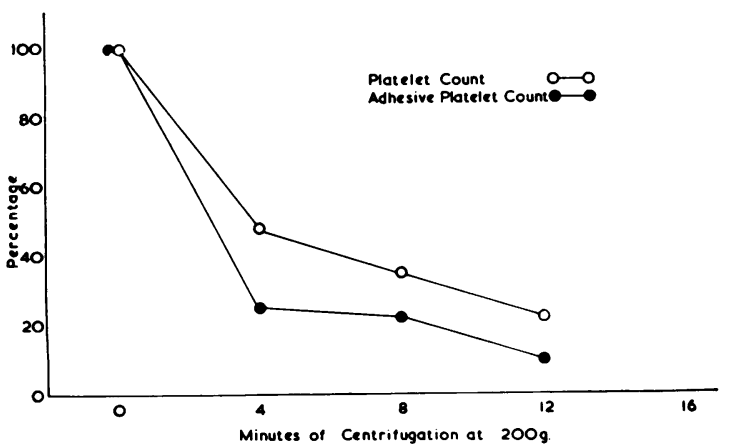

FIG. 3. The total platelet count and adhesive platelet count expressed as a percentage of those values found in plateletrich plasma obtained by sedimentation. The mean values obtained from 10 splenectomized individuals are shown.

VENOUS THROMBOSIS The general pattern of results is similar to that found in the post-splenectomy group. The major and important difference is that in this group the platelet count was within the generally accepted normal range. After only four minutes of centrifugation adhesiveness measured in plateletrich plasma was significantly less than that measured in whole blood or plasma obtained by sedimentation (Table III). In spite of the difference in total platelet count and adhesive platelet count, platelets from patients with venous thrombosis behave similarly to platelets from splenectomized subjects in that the adhesive platelets are apparently easily and preferentially removed from platelet-rich plasma by gentle centrifugation (Fig. 4).

\section{DISCUSSION}

The possibility that there might be some relationship between adhesiveness and sedimentation during centrifugation was first suggested by Stormorken, Lund-Riise, and Rørvik (1965). These authors found

TABLE II

MEAN PLATELET COUNT AND PLATELET ADHESIVENESS MEASURED IN WHOLE BLOOD AND IN PLATELET-RICH PLASMA IN 10 PATIENTS AFTER SPLENECTOMY

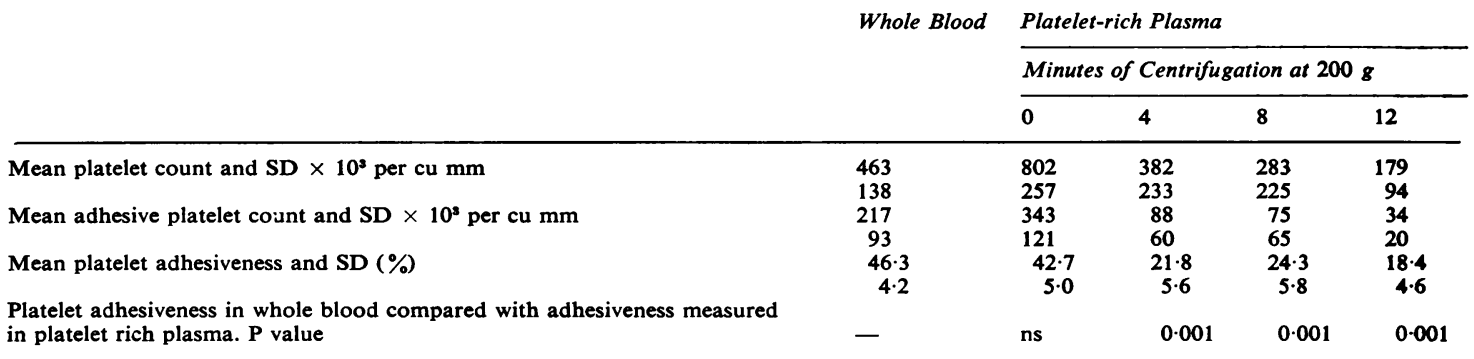

ns $=$ not significant. 


\section{TABLE III}

MEAN PLATELET COUNT AND PLATELET ADHESIVENESS MEASURED IN WHOLE BLOOD AND PLATELET-RICH PLASMA IN FOUR PATIENTS WITH RECURRENT VENOUS THROMBOSIS

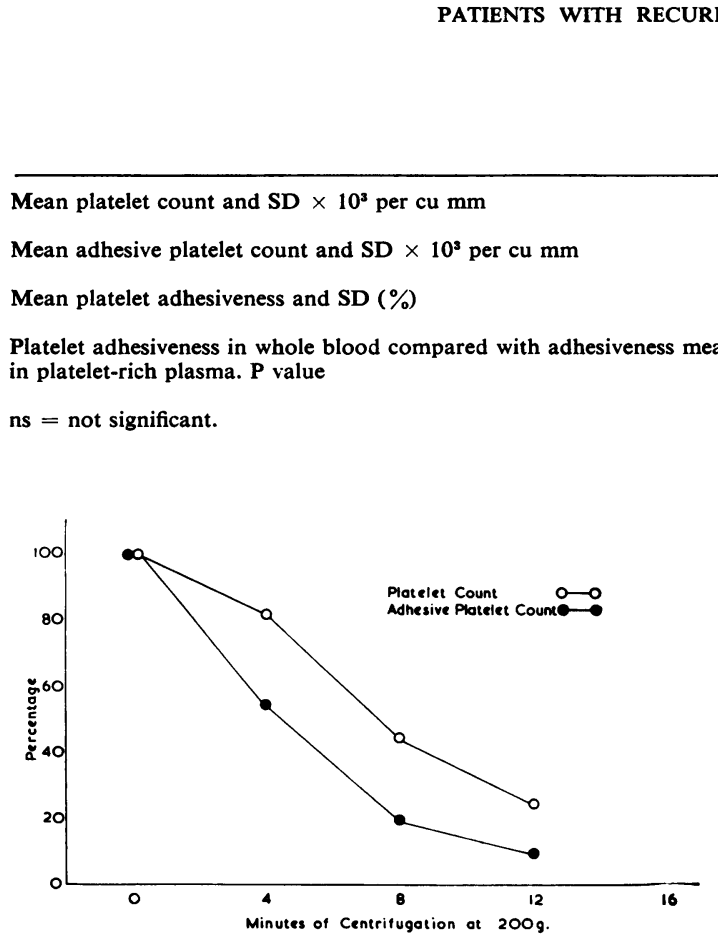

FIG. 4. The total platelet count and adhesive platelet count expressed as a percentage of those values found in plateletrich plasma obtained by sedimentation. The mean values obtained from four individuals suffering from venous thrombosis are shown.

that by halving the volume of citrated blood in the centrifuge tube, thus producing a higher mean ' $g$ ' in the shorter blood columns, and then measuring adhesiveness in the resulting platelet-rich plasma, they were able to reduce the apparent platelet adhesiveness by about $30 \%$. In the present series of experiments the volume of blood in the centrifuge tubes was constant but the time of centrifugation varied.

EFFECT OF CENTRIFUGation The effect of the amount of centrifugation on the preparation of platelet-rich plasma for the measurement of platelet adhesiveness has not previously been reported. In 'normal' individuals, i.e. in those in whom platelet adhesiveness measured in platelet-rich plasma obtained by sedimentation without centrifugation has been within normal limits, the rate of decrease in the total platelet count of platelet-rich plasma is proportional to the duration of centrifugation.

The effect of centrifugation in the preparation of platelet-rich plasma would not matter if nonadhesive and adhesive platelets behaved similarly in a gravity field and their relative proportions did $\stackrel{\circ}{工}$ not change. In the control subjects studied centrifugation had little effect on platelet adhesiveness until the blood had been subjected to $200 \mathrm{~g}$ for eight minutes. Thereafter, adhesiveness progressively de- $\vec{\ominus}$ creased. In the post-splenectomy group, however, there was a marked difference between the behaviour $\square$ of adhesive and non-adhesive platelets. After only four min of centrifugation the total platelet count had been reduced by $54 \%$ but the adhesive platelet count had been reduced by $75 \%$. Platelet adhesiveness measured at this time was approximately half of that measured in plasma obtained by sedimentation (Fig. 3 and Table II). The changes found in the patients with venous thrombosis were similar but slightly less dramatic than those found in the postsplenectomy group. The important difference between these two groups is that the platelet count in the last group was within the normal range.

There are two possible explanations for these findings: either adhesive platelets are physically different from non-adhesive platelets, perhaps being 욱 either larger or heavier and thus sedimenting more $\rightarrow$ rapidly under gravity, or centrifugation causes something to be released from the red cells (? ADP) N which brings about aggregation of some of the platelets present in the specimen. In either case the $\mathcal{O}$ adhesive platelets or platelet aggregates would be spun down with the red cells leaving non-aggregated platelets in suspension in plasma. It is known that 0 the presence of red cells, (Hellem, 1960) or an extract of red cells which contains ADP (Gaarder, $\stackrel{?}{+}$ Jonsen, Laland, Hellem, and Owren, 1961) is necessary before platelets in plasma adhere to glass. Platelets in platelet-rich plasma which does not contain either red cells or ADP do not adhere to glass either in a glass-bead column or in the Wright rotator. 
It has been suggested by Wright (1941) and Hirsh, McBride, and Dacie (1966) that young platelets are more adhesive than old platelets. An increase in the number of young platelets in the blood may be found after splenectomy and in thrombotic states where platelet consumption is increased. In a condition where there is an increase in the young platelet population it may well be that these younger and perhaps stickier platelets can be aggregated by the small quantities of ADP produced by damage to red cells during centrifugation, and it is possible that the number of red cells liable to damage in this way may be increased after splenectomy.

WHOLE BLOOD V PLATELET-RICH PLASMA Platelet adhesiveness measured in platelet-rich plasma after the addition of adenosine diphosphate is very similar to platelet adhesiveness measured in whole blood provided that the platelet-rich plasma has been obtained by sedimentation or centrifugation at a slow speed $(200 \mathrm{~g})$ for eight min or less. After splenectomy, adhesiveness measured in whole blood and in platelet-rich plasma obtained by sedimentation is markedly increased. However, even gentle centrifugation of the blood for four min at $200 \mathrm{~g}$ is enough to reduce platelet adhesiveness in the plasma so obtained by half. Similarly, in patients with venous thrombosis platelet adhesiveness measured in whole blood or in plasma obtained by sedimentation is considerably greater than normal; again, however, gentle centrifugation reduced the apparent adhesiveness significantly. In a study of von Willebrand's disease, Cronberg et al. (1966) showed incidentally that there was little relationship between platelet adhesiveness measured in citrated whole blood and in platelet-rich plasma.

The lack of relationship between platelet adhesiveness measured in whole blood and platelet-rich plasma produced by centrifugation places important limitations on the use of the platelet-rich plasma method in clinical work. This applies even when the platelet-rich plasma is obtained by centrifugation in a standard manner, as platelet adhesiveness measured in plasma may fail to reflect changes which would have been detected had the test been carried out on whole blood.

I am grateful to Professor J. V. Dacie for advice and encouragement, to the physicians and surgeons of Hammersmith Hospital for allowing access to patients under their care, and to Miss Gillian Bolton for valuable technical assistance.

\section{REFERENCES}

Brecher, G., and Cronkite, E. P. (1950). J. appl. Physiol., 3, 365. Caspary, E. A., and Peberdy, M. (1965). Lancet, 1, 1142.

Cronberg, S., Nilsson, I. M., and Silwer, J. (1966). Acta med. scand., $180,43$.

Dacie, J. V., and Lewis, S. M. (1963). Practical Haematology, 3rd ed . Churchill, London.

Gaarder, A., Jonsen, J., Laland, S., Hellem, A., and Owren, P. A. (1961). Nature (Lond.), 192, 531.

Hellem, A. J. (1960). Scand. J. clin. Lab. Invest., 12, suppl. 51.

Horlick, L. (1961). Amer. J. Cardiol., 8, 459.

Hirsch, J., and McBride, J. A. (1965). Brit. med. J., 2, 797.

,-- , and Dacie, J. V. (1966). Ann. Aust. Med., 15, 122. (Stuttg.), 16, 100

O'Brien, J. R. (1961). J. clin. Path., 14, 140.

Reber, K., and Studer, A. (1965). Thrombos. Diathes. haemorrh (Stuttg.), 13, 428.

Stormorken, H., Lund-Riise, Å., and Røvrik, T. O. (1965). Scand. J. clin. Lab. Invest., 17, suppl. 84, 183.

Wright, H. P. (1941). J. Path. Bact., 53, 255. 